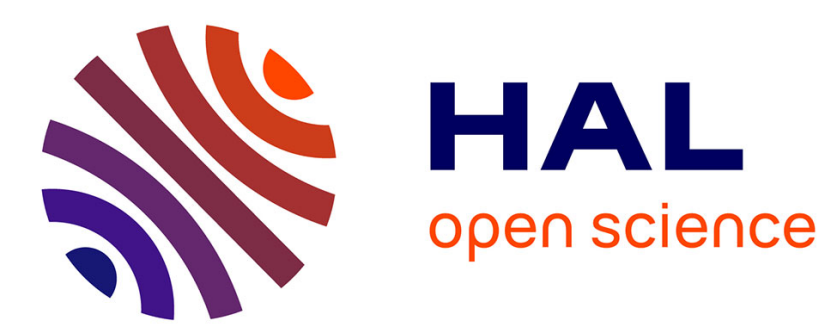

\title{
Evidence of Correlation between Catalyst Particles and the Single-Wall Carbon Nanotube Diameter: A First Step towards Chirality Control
}

\author{
M.-F. C. Fiawoo, Anne Marie Bonnot, H Amara, C Bichara, J \\ Thibault-Pénisson, A Loiseau
}

\section{To cite this version:}

M.-F. C. Fiawoo, Anne Marie Bonnot, H Amara, C Bichara, J Thibault-Pénisson, et al.. Evidence of Correlation between Catalyst Particles and the Single-Wall Carbon Nanotube Diameter: A First Step towards Chirality Control. Physical Review Online Archive (PROLA), 2012, 108 (19), pp.195503. 10.1103/PhysRevLett.108.195503 . hal-01075303

\section{HAL Id: hal-01075303 https://hal.science/hal-01075303}

Submitted on 18 Oct 2014

HAL is a multi-disciplinary open access archive for the deposit and dissemination of scientific research documents, whether they are published or not. The documents may come from teaching and research institutions in France or abroad, or from public or private research centers.
L'archive ouverte pluridisciplinaire HAL, est destinée au dépôt et à la diffusion de documents scientifiques de niveau recherche, publiés ou non, émanant des établissements d'enseignement et de recherche français ou étrangers, des laboratoires publics ou privés. 


\title{
Evidence of Correlation between Catalyst Particles and the Single-Wall Carbon Nanotube Diameter: A First Step towards Chirality Control
}

\author{
M.-F. C. Fiawoo, ${ }^{1,2}$ A.-M. Bonnot,${ }^{3}$ H. Amara, ${ }^{1}$ C. Bichara, ${ }^{4}$ J. Thibault-Pénisson, ${ }^{5}$ and A. Loiseau ${ }^{1}$ \\ ${ }^{1}$ Laboratoire d'Étude des Microstructures, ONERA-CNRS, BP 72, 92322 Châtillon CEDEX, France \\ ${ }^{2}$ Department of Materials Engineering, Monash University, Clayton, VIC 3168, Australia \\ ${ }^{3}$ Institut Louis Néel, CNRS-UJF, BP166, 38042 Grenoble CEDEX 9, France \\ ${ }^{4}$ Centre Interdisciplinaire de Nanoscience de Marseille, CNRS and Aix Marseille University, \\ Campus de Luminy, 13288 Marseille CEDEX 09, France \\ ${ }^{5}$ Institut Matériaux Microélectronique Nanosciences de Provence, \\ CNRS and Aix-Marseille University 13397 Marseille CEDEX 20, France
}

(Received 9 November 2011; published 8 May 2012)

\begin{abstract}
Controlling the structure of single-wall carbon nanotubes during their synthesis by chemical vapor deposition remains a challenging issue. Here, using a specific synthesis protocol and ex situ transmission electron microscopy, we perform a statistical analysis of the structure of the tubes and of the catalyst particles from which they grow. We discriminate two nucleation modes, corresponding to different nanotube-particle junctions, that occur independently of the particle size. With the support of tight binding calculations, we show that a direct control of the nanotube diameter by the particle can only be achieved under growth conditions close to thermodynamic equilibrium.
\end{abstract}

DOI: 10.1103/PhysRevLett.108.195503

PACS numbers: 61.46.Fg, 68.37.Lp

Since their discovery, single-walled carbon nanotubes (SWNTs) have been produced in increasingly larger quantities using catalytic chemical vapor deposition (CCVD). The main hurdle in the development of a SWNT-based technology is to control their structure and more precisely their diameter and chirality. Although post-synthesis sorting techniques are very efficient and useful [1,2], these processes are complex and dramatically damage and shorten the tubes, which can be a serious obstacle for their use in optoelectronic applications. Therefore, it would be highly desirable to control the tube's structure directly during the growth. A limited control of the chiral selectivity has been reported by empirically tuning experimental conditions [3-6], but the reasons for this selectivity are not well understood. This is not surprising as the number of variables involved in the synthesis (e.g., temperature, pressure, catalyst and substrate chemical composition, nature of feedstock) is large. It is a matter of fact that narrowing the nanotube diameter distribution and restricting diameter below $1 \mathrm{~nm}$ leads to a restricted set of chiralities. This has been successfully achieved with the CoMoCAT, a Co-Mo catalytic method that produces SWNTs, process $[3,4]$. According to several authors [7,8] a key parameter is the control of the catalytic nanoparticle (NP) size. However, the relationship between the nanotube diameter and that of the particles is not clear [9]. Transmission electron microscopy (TEM) is the most appropriate technique to analyze this link. Observations can be done either ex situ after the synthesis $[10,11]$ or in situ using environmental TEM [12-14]. A number of ex situ TEM studies addressed the NT-NP diameter issue, showing that the NT diameter is directly related to that of the NP from which it has grown, but with a variable ratio, found to range from 1:1 [11] to 1:1.6 [15] or more [16]. However, in situ experiments have shown nucleation situations where, most often, the NT and NP diameters are not correlated [13]. It should be pointed out, that beside these observations, most of the statistical measurements trying to relate NP and NT diameters were done separately on each population and do not provide a reliable insight on the NP-NT link.

In this Letter, we present a systematic TEM study investigating the correlation between the sizes of the nanotubes or nuclei and of the NP from which they grow, as a function of synthesis time. Ex situ investigation of a large number of samples obtained by stopping the synthesis at different stages was preferred to in situ studies. Indeed, although qualitatively very insightful, environmental TEM experiments focus on one particular event and are not necessarily representative of the general nucleation-growth mechanism. For this ex situ study, we used a procedure described in [17] to allow the direct observation of both NTs and their seed NPs at any stage of the growth without any post-synthesis manipulation. We first show that a threshold (around $5 \mathrm{~nm}$ ) exists in the tube lengths, beyond which tubes can grow very long. We also identify two typical configurations of the graphenic wall of the tubes in contact with the particle (tangential and perpendicular) and show that their relative abundance depends on the synthesis time. With the support of tight binding Monte Carlo (TBMC) simulations, we suggest that tangential growth is favored under slow growth conditions, close to local thermodynamic equilibrium, while the other requires a larger kinetic activation. In the former situation, tube and NP diameters are almost equal, indicating a 
possibility to control the tube diameter via the NPs, a prerequisite for controlling the chiralities.

SWNTs were grown using a hot filament CVD method. Catalyst particles were prepared by dewetting a $1.5 \mathrm{~nm}$ evaporated Co layer on thin $\mathrm{Si}_{3} \mathrm{~N}_{4}$ membranes, in a hydrogen rich atmosphere, during the temperature increase prior to the synthesis. This procedure enabled one to grow NTs directly on electron transparent membranes and to study them in their native state, without any post-synthesis manipulations [17]. The carbon precursor, methane, was introduced $\left(91 \%: 9 \%, \mathrm{H}_{2}: \mathrm{CH}_{4}\right.$ mixture, total pressure $60 \mathrm{mbar}$ ) and, simultaneously, the furnace and filament temperatures were raised to 1073 and $2223 \mathrm{~K}$, respectively. Investigation of the different stages of the NT growth was achieved by stopping the feedstock gases and the heaters at different synthesis times from $40 \mathrm{~s}$ to $30 \mathrm{mn}$. Moreover, the parameters have been tuned to produce SWNTs in low density $\left(<20\right.$ tubes $\left./ \mu \mathrm{m}^{2}\right)$. This allowed us to follow a NT along its entire length up to its bound NP. These synthesis conditions are remarkably suitable for the observation of NPs, nuclei and NTs on their surfaces. TEM observations have been performed with a Philips CM20 operating at $120 \mathrm{kV}$ to reduce the electron irradiation transforming the nuclei under the beam. NTs and NP were observed by transparency through the membrane which is 5 to 10 times thicker and displays a diffraction contrast higher than carbon material. Illumination and focusing conditions were adjusted to get the best contrast on NTs. Nevertheless, images display a strong background due to the membrane. Filtering and image processing techniques have been applied to improve the visibility of the tubes. Both raw data and processed images are shown in the figures. For extracting NTs diameter from images, we used the procedure described in [18] based on an analysis of the contrast profile along a cross section of the tube.

We first determined the population of catalyst NPs and its evolution during the synthesis, by analyzing several hundreds of NPs. As a result of the early introduction of carbon precursor in the reactor, the size distribution almost remains constant, whatever the synthesis time. Indeed, carbonbearing molecules decompose and release carbon at the surface of the NPs as soon as methane is introduced in the reactor (See S1 in the supplemental material [19]). This certainly prevents NPs to coalesce via surface diffusion or Ostwald ripening. In these experiments the samples have a mean NP size between 3.5 and $5.5 \mathrm{~nm}$ with a standard deviation between 1.3 and 2, respectively, (S2, [19]).

We now focus on nuclei. In all cases they are found to be attached by one extremity to a NP. Figures 1(a) and 1(b) shows the most important result of our observations which reveals the existence of two main kinds of nuclei that are either tangential or perpendicular to the NP surface. To distinguish between them, we have defined a somewhat arbitrary criterion. The configuration is considered as perpendicular if the ratio $d_{n} / d_{p}$ is lower than 0.75 , where

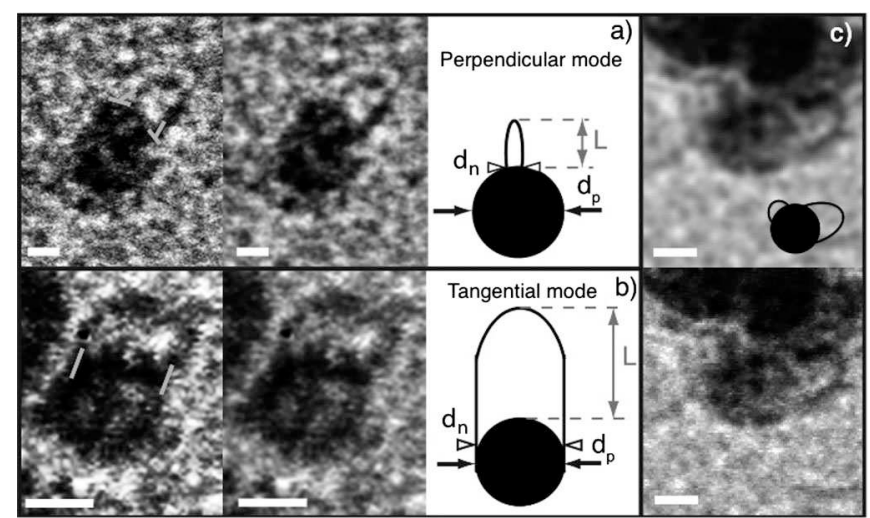

FIG. 1. TEM images of different kinds of nuclei observed and schemes with the dimensions measured $\left(d_{p}\right.$ and $\left.d_{n}\right)$. (a) Perpendicular nucleus type and (b) tangential nucleus type. (c) Several nuclei on a same particle with its corresponding scheme. For all cases, raw image is shown together with a processed image to make the carbon nucleus more visible with respect to the contrast of the amorphous Si-based membrane. Scale bars represent $2 \mathrm{~nm}$.

$d_{n}$ and $d_{p}$ represent the diameters of the nucleus and the NP, respectively. Otherwise, the configuration is assumed to be tangential. The 0.75 ratio threshold has been established in an empirical manner from our statistical observations, taking into account the TEM imaging projection effect which may result from the orientation of the nuclei with respect to the electron beam. The tangential configuration, illustrated in Fig. 1(b), corresponds to the situation commonly observed in CVD samples, with the carbon wall building the tube lying tangentially with respect to the NP surface. In such a case, the resulting NT, if it grows, will have a diameter close to that of its seeding particle, as shown in the example in Fig. 2(a). In the second configuration, shown in Fig. 1(a), the carbon wall is oriented perpendicular to the surface of the NP. In this case, the diameter of the growing tube should be smaller and no longer directly related to that of the NP. This situation is that of the example in Fig. 2(b). Consequently, it often happens that, as in Fig. 1(c), several nuclei of smaller diameters are formed on the same larger diameter NP. The perpendicular configuration has also been observed in the early nucleation stage during in situ TEM experiments [13]
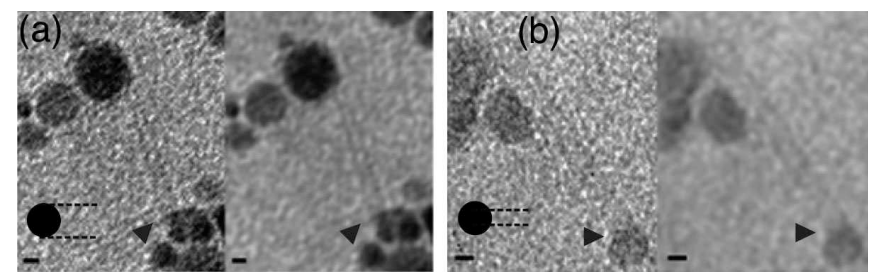

FIG. 2. TEM images of both configurations of nanotubes, tangential (a) and perpendicular (b). From left to right, a growth condition scheme on the image of a nanotube attached to its catalytic nanoparticle, a corresponding Gaussian blurred image. Arrows indicate their supporting particle. Scale bars represent $2 \mathrm{~nm}$. 
and accounts for situations depicted in the literature where NT and NP diameters are not in a ratio close to $1[15,20]$.

To go further, we have investigated the length of the nuclei and the size of all kinds of NPs-active and inactive-, and especially active NPs supporting nuclei and NTs. Figure 3 depicts the situation after 2 min synthesis time, data related to other synthesis times can be seen in S3 in [19]. Whatever the total synthesis time, statistical data show that, whatever its size, a NP can give rise to a nucleus at any moment of the synthesis, and this regardless of the nucleation mode-perpendicular or tangential. In addition, this analysis brings out the existence of a critical nucleus length close to $5 \mathrm{~nm}$. Indeed, as shown in the inset of Fig. 3, whatever the synthesis time, the number of nuclei exceeding $5 \mathrm{~nm}$ in length, is drastically smaller than that of shorter nuclei. The nuclei beyond this critical length are rare, even though they are easier to detect by TEM owing to their significantly longer length (up to $25 \mathrm{~nm}$ ) compared to short nuclei (S3, [19]). In other words, only nuclei of NTs shorter than $5 \mathrm{~nm}$ and long SWNTs ( $>$ $100 \mathrm{~nm}$ ) are observed. This is probably due to a variation in the growth rate, increasing drastically after nanotubes nucleation [12]. Moreover, Hofmann et al. [13] have shown that SWNTs start growing faulty before becoming straight, outlining different growth regimes. This suggests that, in our ex situ observations of quenched samples, the slowest regime is preferentially frozen, yielding a larger fraction of shorter nuclei corresponding to the slowest growth. Consequently, this $5 \mathrm{~nm}$ threshold is assumed to be related to these two phenomena, representing a length transition caused by two different growth regimes. This also means that most of the nuclei observed become inactive just after they appear. Furthermore, while all NPs can give rise to a nucleus that remains below the critical length, in Fig. 3 only those with diameters ranging from about 2 to $4 \mathrm{~nm}$ give birth to longer NTs. Other NPs get deactivated. Different causes can be inferred as discussed in [21,22]. Poisoning by Si from the substrate should be ruled out since most of the

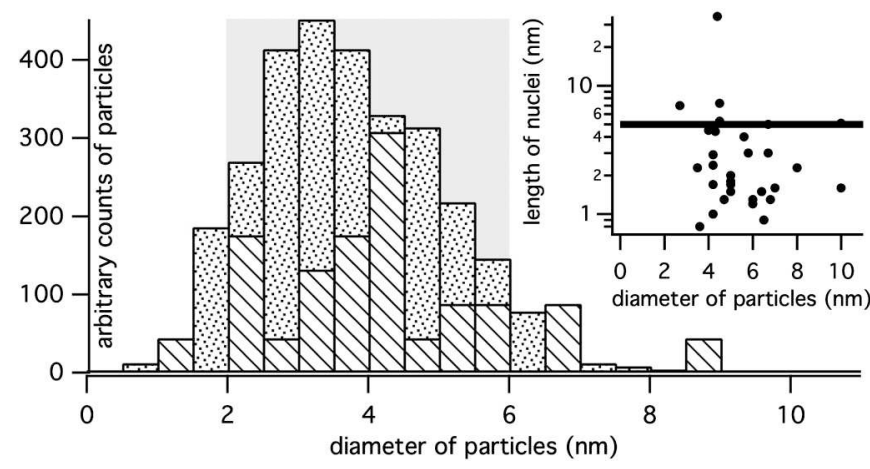

FIG. 3. Histograms of all the particles active (hashed) and inactive (dotted). The gray area highlights the range of particles size favorable for supporting a NT. In inset length of a nucleus vs diameter of the particle supporting the nucleus and the length threshold at $5 \mathrm{~nm}$. particles are found to remain pure Co all along the synthesis (S3, [19]). Also, dissolution in the substrate or Ostwald ripening processes, invoked in [23], are not relevant here since the whole size distribution remains almost constant all along the synthesis (S1-2, [19]). The most probable deactivation cause for NPS smaller than $2 \mathrm{~nm}$ is a too high decomposition rate of carbonaceous molecule with respect to both diffusion and incorporation rates of carbon. As a result these NPs get encapsulated into a carbon shell.

In order to determine the impact of the nucleation mode on the growth, we have examined the configurations of both nuclei and NTs longer than $100 \mathrm{~nm}$, using the criterion defined in Fig. 1 as a function of time. We first found a strong etching effect due to the high fraction of hydrogen in the reactor atmosphere, which causes the destruction of the tubes soon after their growth. Indeed, the nanotube density increases until 2 min synthesis time and then remains constant, meaning the achievement of a steady state regime between nucleation-growth and destruction by etching [24]. This etching effect has two consequences. First metal particles can be continuously reactivated for nucleation, explaining the presence of nuclei all along the synthesis. Second, nuclei and nanotubes present at a given synthesis time reflect the nucleation and growth conditions active at that time.

We then focused on the correlation between nuclei and NTs by distinguishing their type as described in Table I. All along the synthesis, both perpendicular and tangential nuclei and tube configurations are observed. However, the proportion of tangential and perpendicular configurations is found to evolve with synthesis time. At short times ( $40 \mathrm{~s}$ and $2 \mathrm{~min}$ ), perpendicular configurations are dominant, but become a minority upon increasing synthesis times (10 and $30 \mathrm{~min}$ ). This behavior suggests an evolution in the synthesis conditions which may result in a competition between the two nucleation and growth modes.

To get an insight into this competition, we investigate the growth of tube caps sitting on a Ni cluster using atomistic computer simulations based on the tight binding (TB) model we developed for the Ni-C system. This TB model is implemented in a Monte Carlo code working in the grand canonical ensemble (GCMC) [25,26]. Nickel and cobalt have a fairly similar reactivity towards carbon and the computer simulation study described below focuses on

TABLE I. Nuclei and nanotubes at different instant of the synthesis for $\mathrm{Si}_{3} \mathrm{~N}_{4}$. Statistical study is performed on several tens of nano-objects. Values are given in \% (perp. for perpendicular and tang. for tangential).

\begin{tabular}{lccccc}
\hline \hline & \multicolumn{2}{c}{ Nuclei } & & \multicolumn{2}{c}{ Nanotube } \\
\cline { 2 - 3 } \cline { 5 - 6 } Synthesis time & perp. & tang. & & perp. & tang. \\
\hline $40 \mathrm{~s}$ & 65 & 35 & & 75 & 25 \\
$2 \mathrm{~min}$ & 70 & 30 & & 80 & 20 \\
$10 \mathrm{~min}$ & 47 & 53 & & 33 & 67 \\
$30 \mathrm{~min}$ & 45 & 55 & & 30 & 70 \\
\hline \hline
\end{tabular}


generic aspects that are probably valid for both catalysts. The total number of $\mathrm{Ni}$ atoms is kept constant and $\mathrm{C}$ atoms are added or removed with an acceptance probability depending on temperature and on the chemical potential of $\mathrm{C}\left(\mu_{\mathrm{C}}\right)$. To mimic the CVD growth of a tube, $\mathrm{C}$ atoms are tentatively added close to the surface of the Ni catalyst following the Metropolis algorithm [27]. GCMC simulations were performed with starting configurations where different capped $(6,5),(6,6)(10,0)$ and $(9,1)$ SWNT butts with about $80 \mathrm{C}$ atoms are deposited on a $\mathrm{Ni}_{85}$ cluster and subsequently relaxed, as shown for the initial stage of a perpendicular and tangential configuration in Figs. 4(a) and 4(b), respectively. Different situations were observed, depending on the temperature and $\mu_{\mathrm{C}}$ conditions: catalyst encapsulation, tube growth or tube detachment from the $\mathrm{Ni}$ cluster. We focus here on tube growth conditions that correspond to temperatures between 900 and $1060 \mathrm{~K}$ and $\mu_{\mathrm{C}}$ between -7.0 and $-6.5 \mathrm{eV} /$ atom. The carbon chemical potential $\mu_{\mathrm{C}}$ is referred to an ideal gas of monoatomic carbon, explaining its order of magnitude close to the cohesive energy of carbon structures (e.g., $-7.41 \mathrm{eV} /$ atom for graphene in our model). Two typical sequences are presented in Fig. 4, one showing the actual growth of the initial C capped tube [see Fig. 4(b)]. The other showing a growth limited to the right side of the tube where the

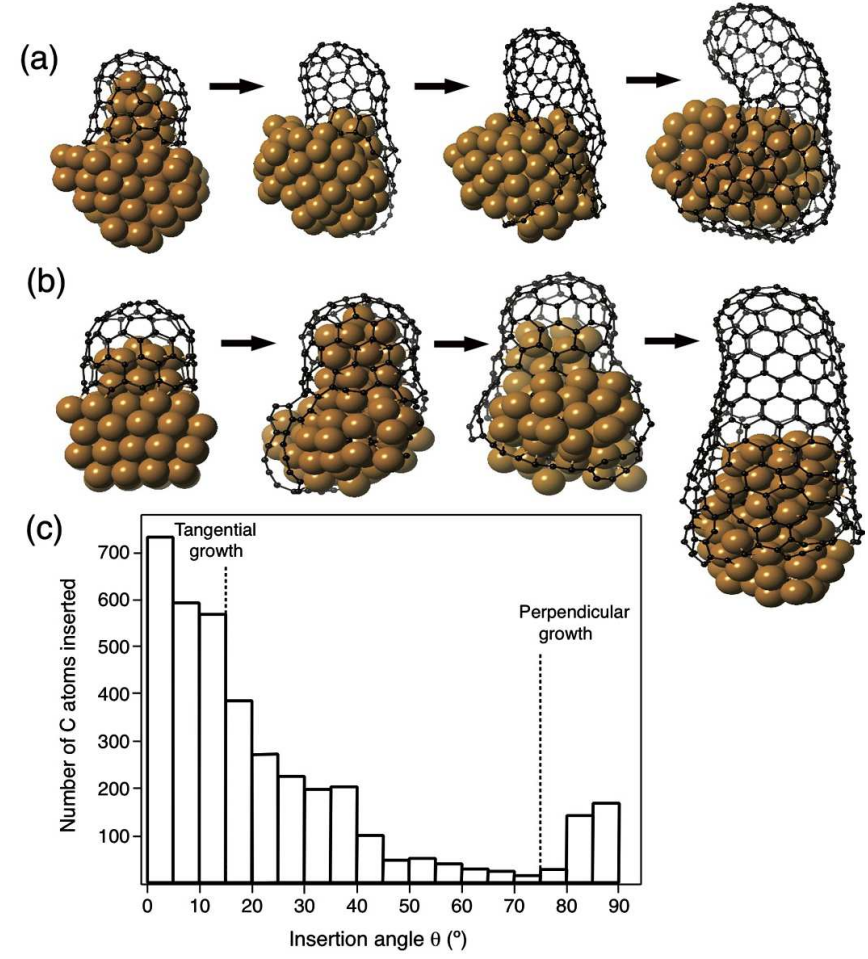

FIG. 4 (color online). (a) Faster tangential growth on the right side and slower perpendicular growth on the left side of the NP, with the tube butt folding over. (b) More efficient tangential growth, with the tube diameter matching the NP size. (c) Histogram of the $\mathrm{C}$ insertion angles (see text) collected on 19 successful growth events. $\mathrm{Ni}$ atoms are displayed in orange, $\mathrm{C}$ in black. graphenic tube wall can grow tangential to the Ni surface, as shown in Fig. 4(a). The left side, anchored perpendicular to the Ni particle surface grows much slower. To rationalize this observation, during the GCMC runs, we record the atomic configurations corresponding to the successful incorporation of a $\mathrm{C}$ atom, $i$. For each such configuration, we calculate the angle $\theta$ between the plane formed by the first three nickel neighbors of $i$ and the line defined by $i$ and its first $\mathrm{C}$ neighbor. In case $i$ has no $\mathrm{C}$ neighbor, the configuration is skipped. $\theta=0^{\circ}$ corresponds to a tangential incorporation while $\theta=90^{\circ}$ corresponds to a perpendicular one. We then make a histogram of $\theta$ values collected on 19 simulations where a growth of the initial carbon cap has been observed. As shown in two typical examples displayed in Fig. 4, most of the successful insertions correspond to $\theta$ close to $0^{\circ}$. Consequently, a succession of such easier incorporations of extra $\mathrm{C}$ atoms at different tangential locations induces the extension of the tube and can be viewed as a preferred growth mechanism at the atomic scale. Interestingly, we note that this easier growth takes place without the carbon lip of the tube pushing on a step edge. As already observed for the nucleation of tube caps in [25] and for graphene growth in [28], we also note that the extension of the tube wall proceeds via carbon chains.

Our simulations suggest to assign the tangential mode to conditions close to equilibrium, and by contrast, the perpendicular mode to conditions driven by kinetics effects, as it involves higher energy barriers and therefore a larger activation energy. It is worth mentioning that the latter has been shown to be the major nucleation mode in high temperature techniques, which are strongly non equilibrium processes and characterized by a high growth rate of a few micron/sec $[10,29]$. Furthermore, in CVD techniques, $d_{n} / d_{p}$ ratio smaller than 1 , assignable to a perpendicular nucleation mode are found for similar growth rates whereas tangential situations correspond to growth rates 2 or 3 order of magnitude lower [12,30]. Turning back to our experiments, it is quite tempting, on the basis of our GCMC simulations, to attribute the evolution in the nucleation mode to a change in the synthesis conditions. Because of the particular synthesis protocol, which favors sudden and rapid condensation of a carbon material at the surface of the particle (S1, [19]), early stages are far from equilibrium conditions and these kinetic conditions favor the perpendicular mode. Once achieved, the steady state regime corresponds to more gentle conditions closer to thermodynamic equilibrium so that tangential mode tends to be dominant.

In summary, we have combined TEM statistical analyses and Monte Carlo simulations to study the link between NPs and SWNTs or their nuclei. We have shown the existence of two nucleation and growth modes. We identified a tangential mode, where the NT diameter is close to that of the particle, and a perpendicular one with the tube smaller than the NP diameter. In our synthesis conditions, both modes are active independently of the NP size, with the perpendicular 
mode dominant at short synthesis time and the tangential one at longer times. We show the existence of a nucleus length threshold, probably correlated to different growth rates. Our TB-GCMC calculations indicate that the tangential incorporation of carbon atoms into the tube structure is the most efficient. On this basis, we propose that a control of the tube chirality should be searched under mild growth conditions, close to thermodynamic equilibrium, as they are the only route towards a direct control of the tube diameter by promoting the tangential growth mode.

The authors acknowledge financial supports from the ACI (Connectubes project), the GDRI-GNT (Graphene and Nanotubes) and the ANR ("SOS Nanotubes", 09-NANO028). We thank O. Stephan and N. Brun from Laboratoire de Physique du Solide-Universite Paris XI-Orsay for the EELS analyses and P. Veyssière for his support.

[1] M. S. Arnold, A. A. Green, J. F. Hulvat, S. I. Stupp, and M. C. Hersam, Nature Nanotech. 1, 60 (2006).

[2] F. Chen, B. Wang, Y. Chen, and L.-J. Li, Nano Lett. 7, 3013 (2007).

[3] S. M. Bachilo, L. Balzano, J. E. Herrera, F. Pompeo, D. E. Resasco, and R. B. Weisman, J. Am. Chem. Soc. 125, 11186 (2003).

[4] B. Wang, L. Wei, L. Yao, L.-J. Li, Y. Yang, and Y. Chen, J. Phys. Chem. C 111, 14612 (2007).

[5] W.-H. Chiang and R. Mohan Sankaran, Nature Mater. 8, 882 (2009).

[6] M. He, A. I. Chernov, V. Fedotov, E. D. Obraztsova, J. Sainio, E. Rikkinen, H. Jiang, Z. Zhu, Y. Tian, E. I. Kauppinen et al., J. Am. Chem. Soc. 132, 13994 (2010).

[7] C. L. Cheung, A. Kurtz, H. Park, and C. M. Lieber, J. Phys. Chem. B 106, 2429 (2002).

[8] G. H. Jeong, S. Suzuki, Y. Kobayashi, A. Yamazaki, H. Yoshimura, and Y. Homma, Appl. Phys. Lett. 90, 043108 (2007).

[9] D. Schebarchov, S. C. Hendy, E. Ertekin, and J.C. Grossman, Phys. Rev. Lett. 107, 185503 (2011).

[10] J. Gavillet, J. Thibault, O. Stéphan, H. Amara, A. Loiseau, Ch. Bichara, J.-P. Gaspard, and F. Ducastelle, J. Nanosci. Nanotechnol. 4, 346 (2004).
[11] Y. Li, W. Kim, Y. Zhang, M. Rolandi, D. Wang, and H. Dai, J. Phys. Chem. B 105, 11424 (2001).

[12] M. Lin, J. P. Y. Tan, C. Boothroyd, K. P. Loh, E. S. Tok, and Y.-L. Foo, Nano Lett. 6, 449 (2006).

[13] S. Hofmann, R. Sharma, C. Ducati, G. Du, C. Mattevi, C. Cepek, M. Cantoro, S. Pisana, A. Parvez, F. CervantesSodi et al., Nano Lett. 7, 602 (2007).

[14] H. Yoshida, S. Takeda, T. Uchiyama, H. Kohno, and Y. Homma, Nano Lett. 8, 2082 (2008).

[15] A. G. Nasibulin, P. V. Pikhitsa, H. Jiang, and E. I. Kauppinen, Carbon 43, 2251 (2005).

[16] C. Lu and J. Liu, J. Phys. Chem. B 110, 20254 (2006).

[17] M.-F. Fiawoo, A.-M. Bonnot, V. Jourdain, T. Michel, M. Picher, R. Arenal, J. Thibault-Pénisson, and A. Loiseau, Surf. Sci. 603, 1115 (2009).

[18] R. Fleurier, J.-S. Lauret, U. Lopez, and A. Loiseau, Adv. Funct. Mater. 19, 2219 (2009).

[19] See Supplemental Material at http://link.aps.org/ supplemental/10.1103/PhysRevLett.108.195503 for details.

[20] T. Hiraoka, S. Bandow, H. Shinohara, and S. Iijima, Carbon 44, 1853 (2006).

[21] A. A. Puretzky, D.B. Geohegan, X. Fan, and S.J. Pennycook, Appl. Phys. Lett. 76, 182 (2000).

[22] M. Picher, E. Anglaret, R. Arenal, and V. Jourdain, Nano Lett. 9, 542 (2009).

[23] M. Picher, E. Anglaret, R. Arenal, and V. Jourdain, ACS Nano 5, 2118 (2011).

[24] Etching is suppressed when adding, below the Co film, a thin Ti film which is known for its hydrogen adsorption ability. As a result, the nanotube density is no longer constant but dramatically increases as a function of synthesis time (S4 in [19]).

[25] H. Amara, C. Bichara, and F. Ducastelle, Phys. Rev. Lett. 100, 056105 (2008).

[26] H. Amara, J.-M. Roussel, C. Bichara, J.-P. Gaspard, and F. Ducastelle, Phys. Rev. B 79, 014109 (2009).

[27] D. Frenkel and B. Smit, Understanding Molecular Simulation (Academic Press, London, 1996).

[28] E. Loginova, N. C. Bartelt, P. J. Feibelman, and K. F. McCarty, New J. Phys. 10, 093026 (2008).

[29] A. A. Puretzky, H. Schittenhelm, X. Fan, M. J. Lance, L. F. Allard, Jr, and D. B. Geohegan, Phys. Rev. B 65, 245425 (2002).

[30] D. B. Geohegan, A. A. Puretzky, I. N. Ivanov, S. Jesse, G. Eres, and J. Y. Howe, Appl. Phys. Lett. 83, 1851 (2003). 